\title{
Amlexanox, a selective inhibitor of IKBKE, generates anti-tumoral effects by disrupting the Hippo pathway in human glioblastoma cell lines
}

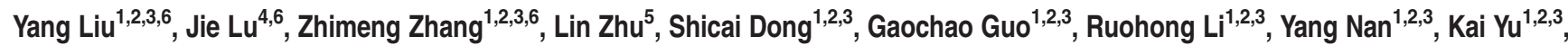 \\ Yue Zhong ${ }^{1,2,3}$ and Qiang Huang ${ }^{*, 1,2,3}$
}

\begin{abstract}
Glioblastoma multiforme (GBM) is the most prevalent form of malignant brain tumor. Amlexanox, a novel compound, has been shown to have anti-cancer potential. In this study, the anti-tumoral effects and the underlying mechanisms of amlexanox were investigated. Amlexanox significantly suppressed proliferation and invasion and induced apoptosis in glioblastoma cells. Furthermore, we found that amlexanox altered the protein expression of the Hippo pathway by downregulating IKBKE. Our data indicates that IKBKE directly targets LATS1/2 and induces degradation of LATS1/2, thereby inhibiting the activity of the Hippo pathway. In vivo results further confirmed the tumor inhibitory effect of amlexanox via the downregulation of IKBKE, and amlexanox induced no apparent toxicity. Collectively, our studies suggest that amlexanox is a promising therapeutic agent for the treatment of GBM.
\end{abstract}

Cell Death and Disease (2017) 8, e3022; doi:10.1038/cddis.2017.396; published online 31 August 2017

GBM is the most common and most malignant tumor in the central neural system. ${ }^{1}$ The biological properties of GBM are strong proliferation, rapid migration, intensive invasion, genetic alteration and increased angiogenesis. ${ }^{2}$ Although there have been great advances in neuroimaging, neurosurgical techniques, radiotherapy and chemotherapy, the overall survival for patients with GBM has still remained unchanged for the last several decades. ${ }^{3,4}$ The median survival time of GBM patients is currently 15-17 months from diagnosis, which is only a few months longer than 30 years ago. The 5-year overall survival rate of GBM is $\sim 5.1 \%$. $^{5,6}$ Recently, an increasing number of studies have been focusing on the identification of novel molecular targets that are crucial for developing more effective therapies. ${ }^{7}$ IKBKE (inhibitor of nuclear factor kappa-B kinase subunit epsilon, also called IKK or IKK-i), which is a member of the $I_{\kappa B}$ kinase (IKK) family, has been identified as an oncogenic protein and found to be upregulated in breast cancer, ${ }^{8}$ ovarian cancer, ${ }^{9}$ prostate cancer $^{10}$ and glioma. ${ }^{2,11}$ Over-expression of IKBKE inhibited glioma cell apoptosis and, in contrast, siRNA-mediated suppression of IKBKE increased the sensitivity of glioma cells to apoptotic inducers. ${ }^{11}$ Our previous study indicated that IKBKE is an emerging key regulator of the malignant progression of gliomas, which is associated with a poor prognosis, and silencing of IKBKE by siRNA can reduce the proliferative and invasive abilities of glioma cells, highlighting it as a potential therapeutic target. ${ }^{2}$

Amlexanox is an anti-inflammatory, anti-allergic, immunomodulator and has been developed for the treatment of ulcers, allergic rhinitis and asthma in the clinic. ${ }^{12}$ Recently, amlexanox was found to be an inhibitor of the protein kinases TBK1/
IKBKE and improve obesity related metabolic dysfunction. ${ }^{13}$ In the present study, we identified amlexanox as a potent antiglioma drug by downregulating IKBKE.

The Hippo pathway is an evolutionarily conserved regulator of tissue and organ growth and controls multiple cellular functions that are central to tumorigenesis, including proliferation and apoptosis. ${ }^{14}$ The Hippo pathway comprises the STE20-like protein kinase1 (MST1, also known as STK4) and MST2 (also known as STK3), large tumor suppressors 1 and 2 (LATS1/2), MOB kinase activator (MOB1A/B), SAV1 and neurofibromatosis 2 (NF2). When the Hippo pathway is activated, MST1/2 phosphorylate and activate LATS1/2 with the help of SAV1. Then, LATS1/2 phosphorylate and restrict the activity of two transcriptional co-activators, Yes-associated protein (YAP) and transcriptional coactivator with a PDZbinding motif (TAZ). ${ }^{15,16}$ The Hippo pathway regulates the nuclear translocation of YAP1, which is an important downstream player, and has been found to have a possible link with stem/progenitor cells, organ size and cancer. ${ }^{17}$ YAP1 has been implicated as an oncoprotein and may promote glioblastoma growth, and high levels of expression has been associated with clinically aggressive glioblastoma subtypes. ${ }^{18}$ We investigated the mechanism in which IKBKE may function as a negative regulator of the Hippo pathway through controlling LATS1/2 stability and YAP1 activity.

In this study, we demonstrated the effects of amlexanox on the migration and invasion of glioblastoma cells through the downregulation of IKBKE levels, which ultimately targets the Hippo pathway. Amlexanox is a potentially attractive antineoplastic agent that might benefit therapy for GBM in the future.

\footnotetext{
${ }^{1}$ Department of Neurosurgery, Tianjin Medical University General Hospital, Tianjin, China; ${ }^{2}$ Key Laboratory of Post-Trauma Neuro-Repair and Regeneration in Central Nervous System, Ministry of Education, Tianjin, China; ${ }^{3}$ Tianjin Key Laboratory of Injuries, Variations and Regeneration of Nervous System, Tianjin, China; ${ }^{4}$ Department of Neurosurgery, Shandong Provincial Qianfoshan Hospital, Shandong, China and ${ }^{5}$ Department of Pathology, The Second People's Hospital of Liaocheng, Linqing, China *Corresponding author: Q Huang, Department of Neurosurgery, Tianjin Medical University General Hospital, 154 Anshan Road, Heping District, Tianjin 300052, China. Tel/Fax: +86 13820689221; E-mail: huangqiang209@163.com

${ }^{6}$ These authors contributed equally to this work.

Received 24.5.17; revised 25.6.17; accepted 02.7.17; Edited by A Stephanou
} 
a

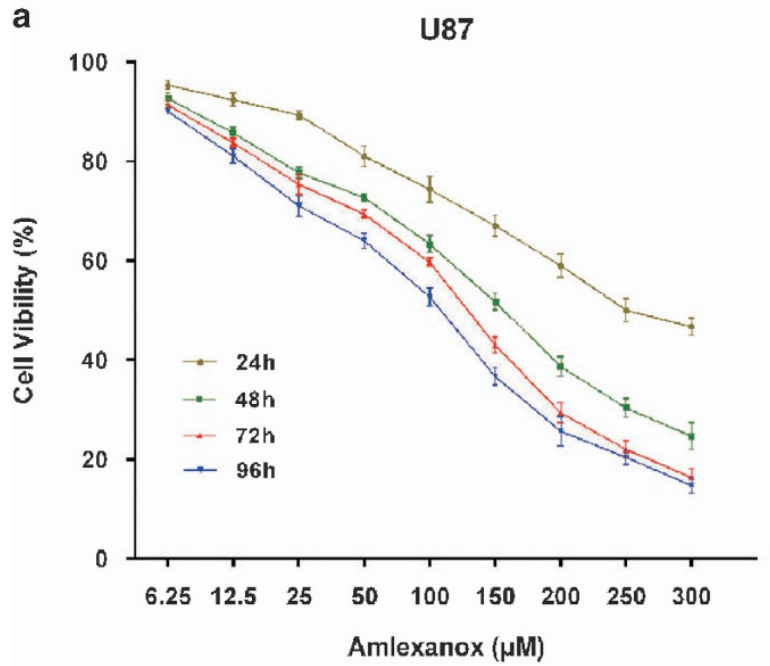

C
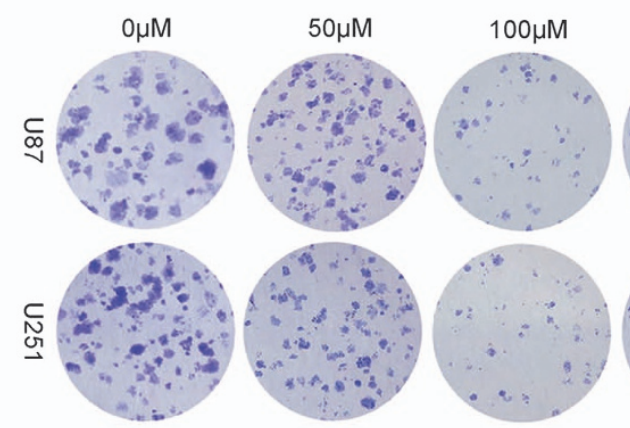

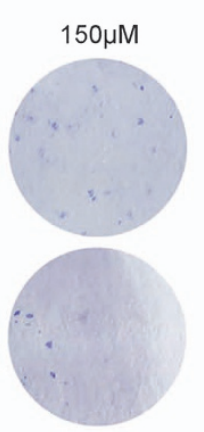

Figure 1 Amlexanox inhibited proliferation and colony formation of glioma cells. (a and $\mathbf{b})$ U87 and U251 cells were treated with amlexanox (0-300 $\mu \mathrm{M})$ for $24,48,72$ and $96 \mathrm{~h}$, and cell vibility was detected using CCK-8 assay. (c) U87 and U251 cells were treated with various concentrations of amlexanox (0,50,100 and 150 $\mu$ M) for 2 weeks. (d) The cell colonies were stained and quantified, and the results are presented in the histogram. The results shown are representative of at least three independent experiments. Data are shown as the mean \pm S.D. ${ }^{*} P<0.05$ and ${ }^{\star *} P<0.01$ compared with the control group

\section{Results}

Amlexanox suppressed GBM cells proliferation and induced G0/G1 phase arrest. To investigate the effects of amlexanox on the proliferation of glioma cells in vitro, the viability rate of U87 and U251 was assessed by CCK-8. Supplementary Figure 1 shows amlexanox chemical structure. Treatments of amlexanox inhibited cell viability in a dose- and time-dependent manner at different concentrations $(6.25,12.5$, $25,50,100,150,200,250$ and $300 \mu \mathrm{M}$ ) for 24-96 h compared with control cells (Figures 1a and b). The IC50 values for $72 \mathrm{~h}$ of amlexanox treatment were approximately $140 \mu \mathrm{M}$ in U87 and $120 \mu \mathrm{M}$ in U251 cells. Based on the IC50 measured in the study, concentrations of 50,100 and $150 \mu \mathrm{M}$ were selected to be used in the following experiments. Furthermore, the ability of both glioma cell lines to form colonies was evaluated with or without amlexanox treatment for a period of 2 weeks (Figure 1c). The results showed that colony formation rates were significantly reduced in the amlexanox-treated groups in both glioma cell lines (Figure 1d).

The effect of amlexanox on the cell cycle progression of glioma cells was evaluated by DNA content analysis. U87 and U251 cells were treated with various concentration of amlexanox for $72 \mathrm{~h}$. Amlexanox induced cell cycle arrest in a b
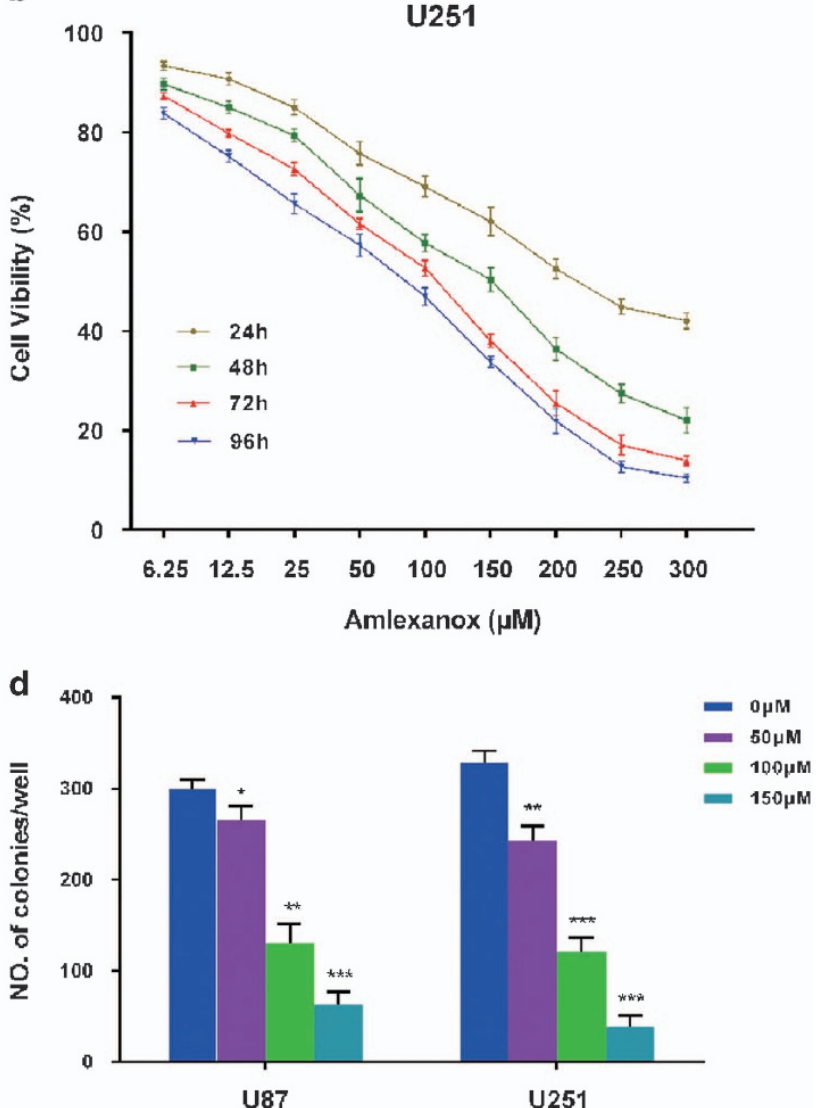
a

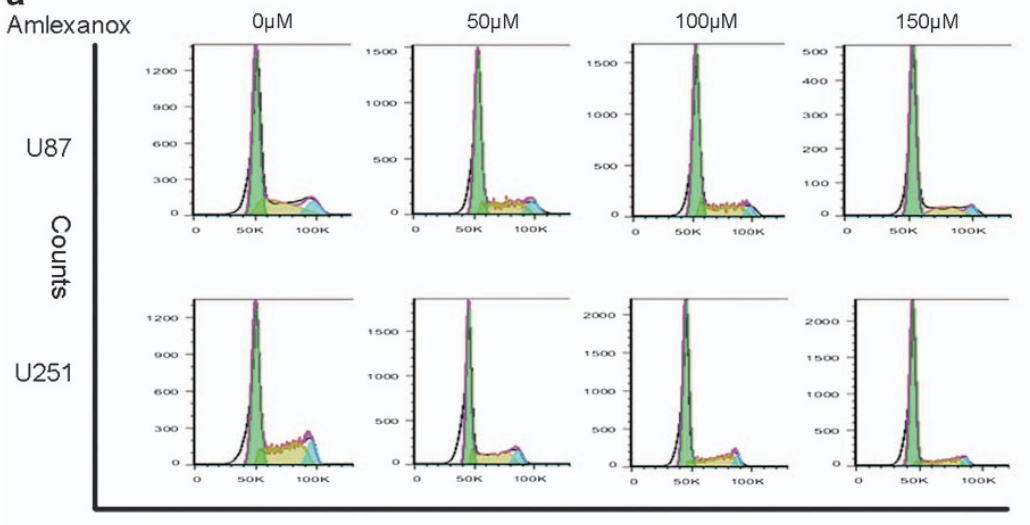

$\mathrm{PI}$

C

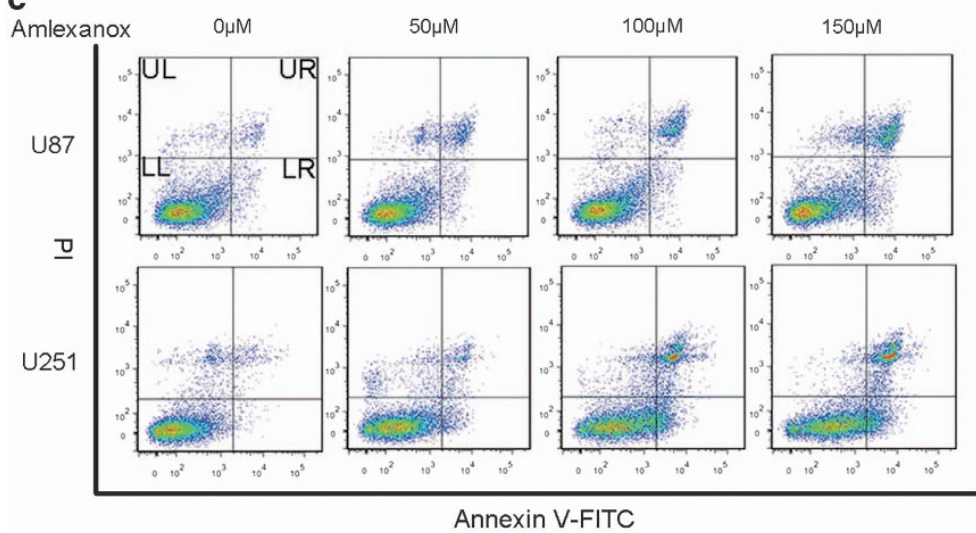

b

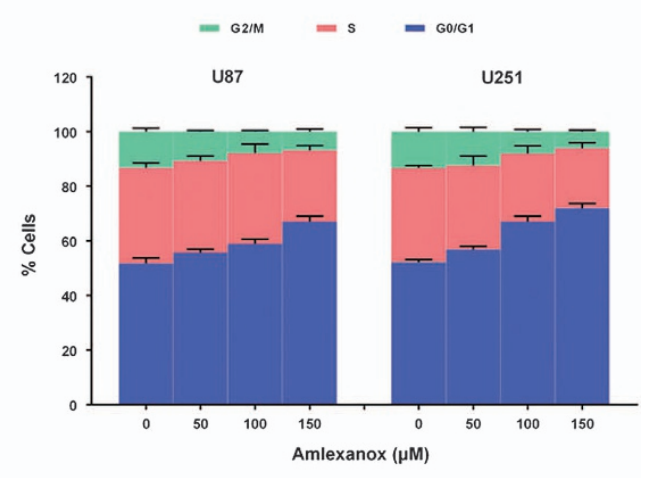

d

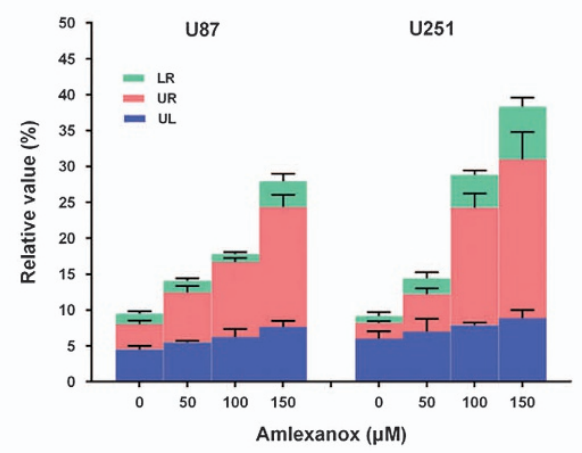

Figure 2 Amlexanox induced G0/G1 phase arrest and apoptosis in glioma cells. (a) U87 and U251 cells were treated with various concentrations of amlexanox for $72 \mathrm{~h}$ and then, assessed by propidium iodide staining-coupled flow cytometry. (b) Bar graphs showing a concentration-dependent cell cycle arresting effect of amlexanox in U87 and U251 cells. (c) After treatment with various concentrations of amlexanox for $72 \mathrm{~h}$, U87 and U251 cells were stained with annexin V-FITC and PI for analysis using flow cytometry. (d) Increased apoptotic cells in U87 and U251 were quantified and presented in the histogram. Data are shown as the mean \pm S.D. $n=3$ for each group. ${ }^{*} P<0.05$ and ${ }^{* *} P<0.01$ compared with the control group

healing assay and transwell assay. The results of the wound healing assay showed that healing decreased gradually with increased concentrations of amlexanox (Figures $3 a$ and b). Amlexanox at $150 \mu \mathrm{M}$ significantly reduced the migration capability of U87 and U251 cells compared with that of control. The results of the wound healing assay were also supported by that of the transwell migration assay (Figures $3 \mathrm{c}$ and d).

Amlexanox impaired the invasion of U87 and U251 cells across transwell chambers compared with that observed in control (Figures $3 e$ and f). Matrix metalloproteinases (MMPs), such as MMP-2 and MMP-9, play important roles in the invasion and malignancy of glioma cells. ${ }^{19}$ After U87 and U251 cells were treated with amlexanox at various concentrations for $72 \mathrm{~h}$, the protein expression levels of MMP-2 and MMP-9 were checked by western blot (Figure $3 \mathrm{~g}$ ). The results showed that amlexanox inhibited the expression of MMP-2 and MMP-9 in a dose-dependent manner, and $150 \mu \mathrm{M}$ amlexanox significantly inhibited the expression of MMP-2 and MMP-9 compared with that in control.

Amlexanox reverted the malignant phenotype of GBM cells via activating the Hippo pathway mediated by downregulation of IKBKE. Amlexanox has been shown to block IKBKE activity by interacting with the enzyme in the ATP-binding region, and amlexanox treatment reduced IKBKE protein levels in brown adipose tissue and non-small cell lung cancer cells. ${ }^{13,20}$ Since we have observed the inhibitory effect of amlexanox on glioma cells, we next investigated the effect of amlexanox on molecular signals in the U87 and U251 cell lines, including the Hippo pathway. The Hippo pathway controls tissue growth and cell fate, whereas pathway deregulation can induce tumors in model organisms and occurs in a broad range of human carcinomas. ${ }^{14}$ We found that amlexanox treatment could inhibit the IKBKE protein and alter the abundance and phosphorylation status of the Hippo pathway proteins in glioma cells. As shown in Figures $4 a$ and $b$, first, we examined the level of IKBKE using western blot and found that there was a significant decrease in IKBKE expression in response to amlexanox treatment. However, the qRT-PCR results showed that the IKBKE mRNA level remained unchanged after amlexanox treatment, suggesting that amlexanox did not affect IKBKE transcription (Supplementary Figures 2a-d).

Compared with the levels in the control cells, amlexanox treatment increased the level of LATS1/2 and phospho-YAP1 
a
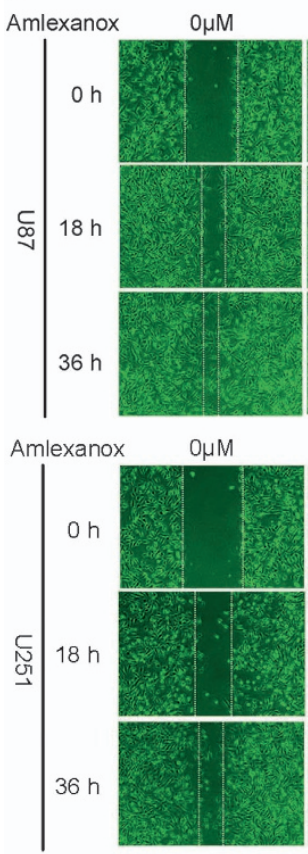

。

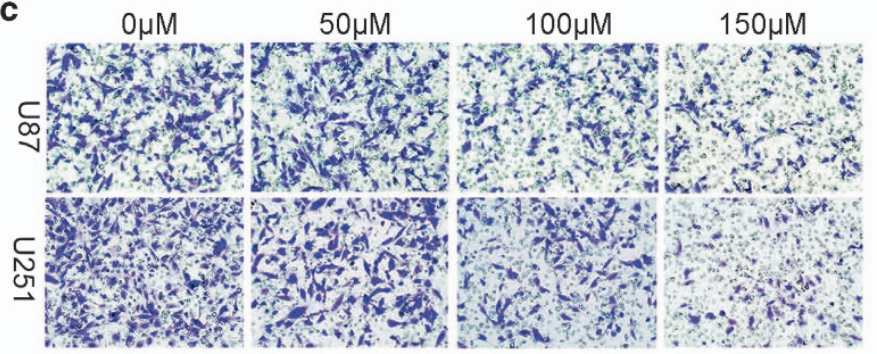

e

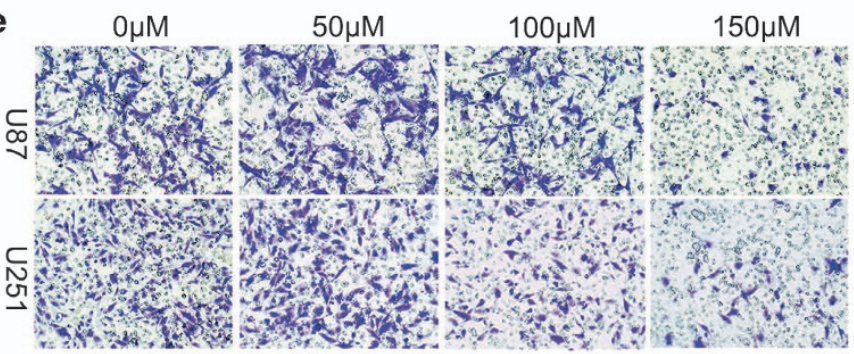

g

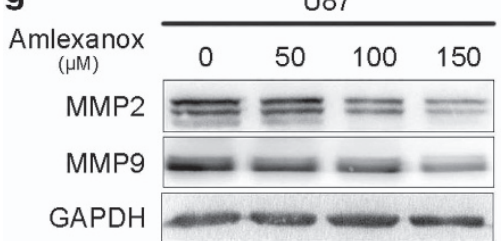

$100 \mu \mathrm{M}$

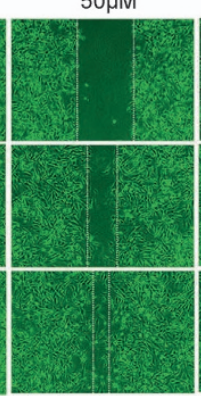

$50 \mu \mathrm{M}$
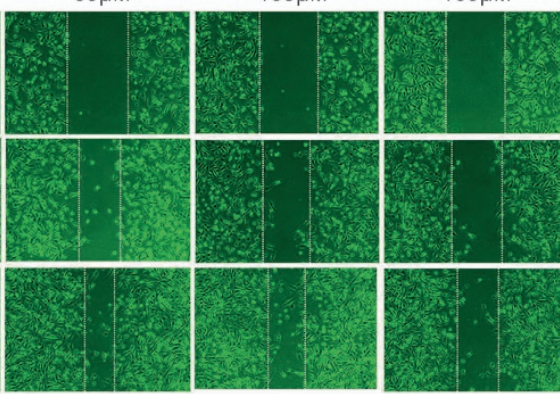

$150 \mu \mathrm{M}$

$150 \mu \mathrm{M}$

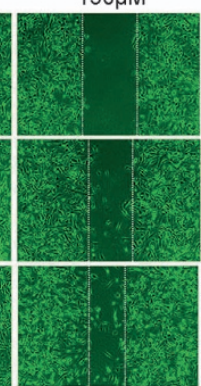

$150 \mu \mathrm{M}$
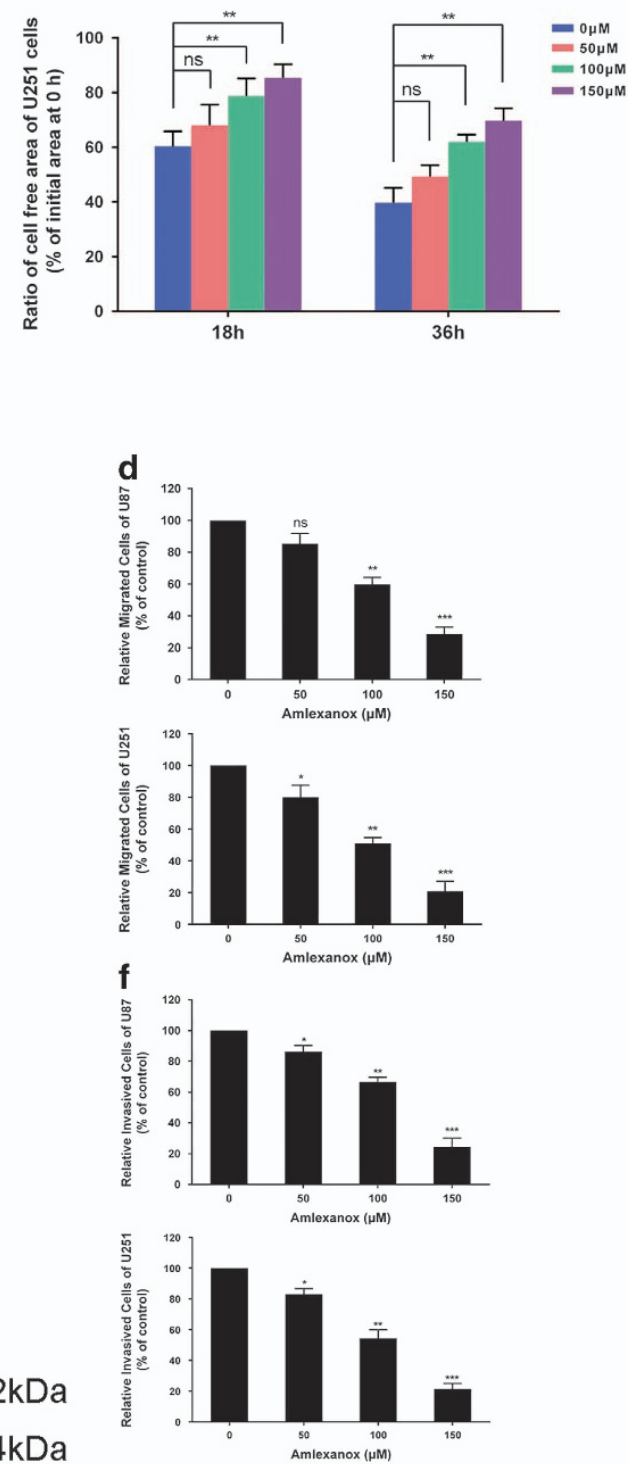

Figure 3 Amlexanox inhibited migration and invasion of glioma cells. U87 and U251 cells were treated with amlexanox at various concentrations of $0,50,100$ and $150 \mu \mathrm{M}$ for $72 \mathrm{~h}$. (a) Representative images from the wound healing assay of each cell are displayed for 18 or $36 \mathrm{~h}$ after cell seeding. (b) The results of the wound healing were quantified and presented in the histogram. (c) Representative images of the transwell migration assay (without Matrigel) are shown for $12 \mathrm{~h}$ after U87 cells seeding and $24 \mathrm{~h}$ after U251 cells seeding. (d) Statistical analysis of migrating cell numbers. (e) Representative images of the transwell invasion assay (with Matrigel) are shown for $24 \mathrm{~h}$ after U87 cells seeding and $48 \mathrm{~h}$ after U251 cells seeding. (f) Statistical analysis of migrating cell numbers. (g) The protein levels of MMP-2 and MMP-9 were evaluated by western blot. The results shown are representative of at least three independent experiments. Data are shown as the means \pm S.D. ${ }^{*} P<0.05$ and ${ }^{* *} P<0.01$ compared with the control group 
a

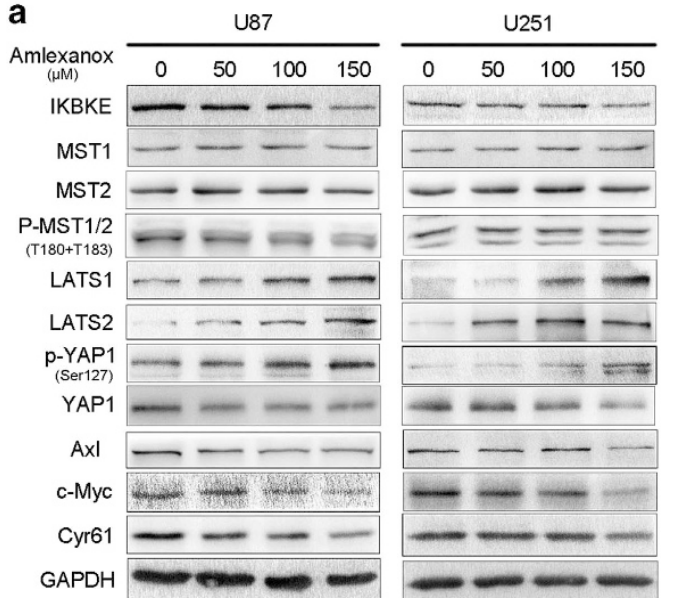

b

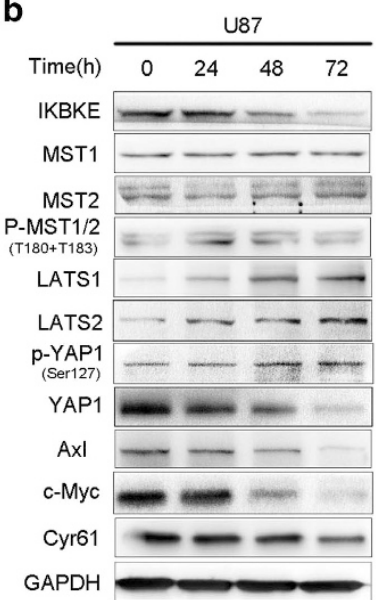

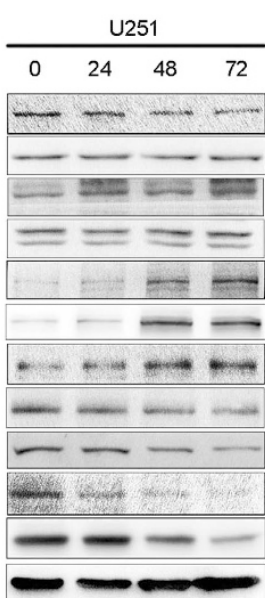

$80 \mathrm{kDa}$

$59 \mathrm{kDa}$

$56 \mathrm{kDa}$

$56 \mathrm{kDa}$

$140 \mathrm{kDa}$

$140 \mathrm{kDa}$

$70 \mathrm{kDa}$

$70 \mathrm{kDa}$

$95 \mathrm{kDa}$

$60 \mathrm{kDa}$

$41 \mathrm{kDa}$

$37 \mathrm{kDa}$

Figure 4 Amlexanox reduced the protein expression of the Hippo pathway through downregulation of IKBKE. (a) U87 and U251 cells were treated with various concentrations of amlexanox for $72 \mathrm{~h}$. The cells were harvested, and the effects of amlexanox on the protein expression of the Hippo pathway were detected by western blot. (b) U87 and U251 cells were treated with amlexanox at $150 \mu \mathrm{M}$ for 24,48 and $72 \mathrm{~h}$. Cell lysates were collected, and the protein expression were detected by western blot as in (a)

(Ser127), whereas YAP1 and the downstream targeted effectors of the Hippo pathway, including Axl, c-Myc and Cyr61, ${ }^{21}$ were significantly reduced in a dose-and timedependent manner. In contrast, the levels of protein for MST1/2 and phospho-MST1/MST2 (Thr183 in MST1 and Thr180 in MST2) did not change after amlexanox treatment.

IKBKE inactivates the Hippo pathway by downregulation of LATS1/2. Next, we identified the potential molecular mechanisms by which IKBKE affects the Hippo pathway. The results showed that IKBKE knockdown in U87 and U251 cells dramatically elevated LATS1/2 and phosphoYAP1 (Ser127) proteins and suppressed YAP1 protein and the downstream targets of the Hippo pathway, while nearly had no effect on the expression of MST1/2 and phosphoMST1/MST2 (Thr183 in MST1 and Thr180 in MST2) proteins (Figure 5a). In contrast, the over-expression of IKBKE in U87 and U251 cells decreased LATS1/2 and phospho-YAP1 (Ser127) protein expression and increased the expression of YAP1 proteins, whereas it similarly did not change the levels of MST1/2 and phospho-MST1/MST2 (Thr183 in MST1 and Thr180 in MST2) proteins (Figure 5b), suggesting the very possible involvement of the Hippo pathway in IKBKEmediated glioma growth and that IKBKE could regulate LATS1/2 and YAP1 in the pathway.

As shown in Figures $5 \mathrm{c}$ and $\mathrm{d}$, compared to the control, the downregulation of IKBKE triggered a reduction of YAP1 in the total and nuclear protein extracts of U87 and U251 cells, but not in the cytoplasm. In addition, the immunofluorescence analysis further confirmed the change in the cytoplasm and nucleus localization of YAP1 when IKBKE was silenced (Figure 5e; Supplementary Figures $3 a$ and $b$ and b). YAP1 phosphorylation on serine 127 is a direct target of LATS1/2 kinase. $^{22}$ Phosphorylation of YAP1-Ser127 by LATS1/2 generates a 14-3-3 binding site and induces YAP1 cytoplasmic retention and degradation. ${ }^{23,24}$
IKBKE downregulates LATS1/2 by promoting their degradation. We further found that there was no significant difference between the control and shIKBKE groups for LATS1/2 mRNA expression, which was determined by qRTPCR (Figure 6a), indicating that IKBKE may function as an endogenous regulator of LATS $1 / 2$ at the post-translational level.

To explore the interaction between IKBKE and LATS1/2, we performed Co-IP experiments with the IKBKE and LATS1/2 antibody. The result indicated the existence of combination between IKBKE and LATS1/2 in glioma cells (Figure 6b). It has been reported that LATS2 destabilization is mainly regulated by proteasome and ubiquitin pathway. ${ }^{22,25,26}$ After treatment with the proteasome inhibitor MG132, we observed that the IKBKE-induced reduction of LATS2 expression was blocked (Figure 6c). we next analyzed LATS2 ubiquitination levels in IKBKE knockdown U87 cells. IKBKE knockdown decreased LATS2 ubiquitination, indicating that IKBKE may regulate LATS2 stability through promoting its polyubiquitin degradation (Figure 6d). To measure the kinetics of LATS2 degradation, we used cycloheximide $(\mathrm{CHX})$ to inhibit protein synthesis. The LATS2 protein degraded faster in the control cells than in the shIKBKE cells (Figure 6e). Since IKBKE interacted with both LATS1 and LATS2, which are homologous to each other, ${ }^{27}$ suggesting that IKBKE may be involved in the regulation of LATS1 through identical mechanisms.

Taken together, the results indicated that IKBKE may function as a negative regulator of the Hippo pathway through controlling LATS1/2 stability and YAP1 activity.

Amlexanox suppresses glioblastoma growth in xenograft models. To investigate the potential effects of amlexanox on glioblastoma growth in vivo, we first established a subcutaneous glioma model using U87 cells as described previously. Seven days after implantation, DMSO or amlexanox were intraperitoneally injected every day for 21 days. Amlexanox treatment exhibited an inhibitory effect on subcutaneous tumor growth and significantly decreased the tumor volume compared with that observed in the control group on day 21 
a

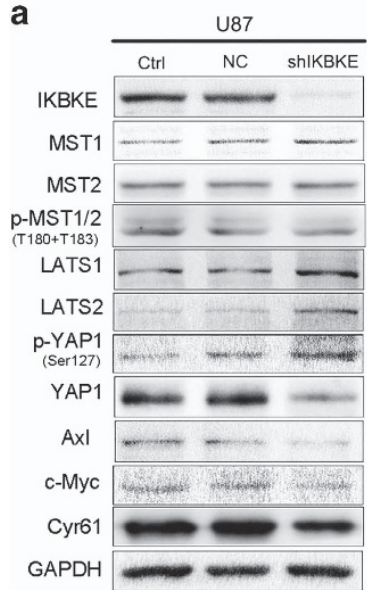

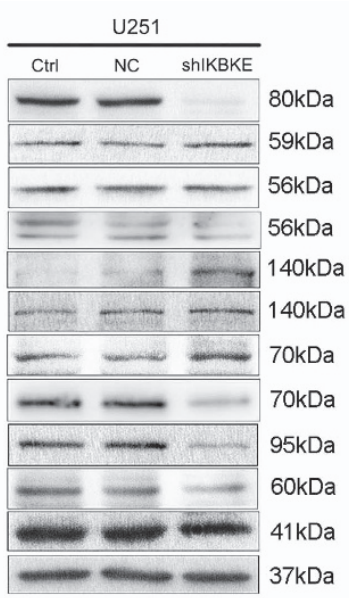

U87

C

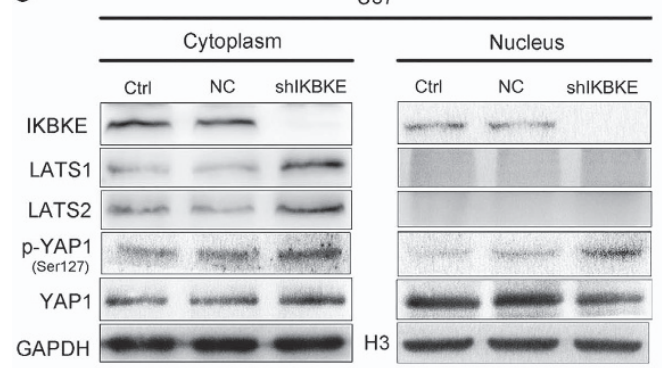

d

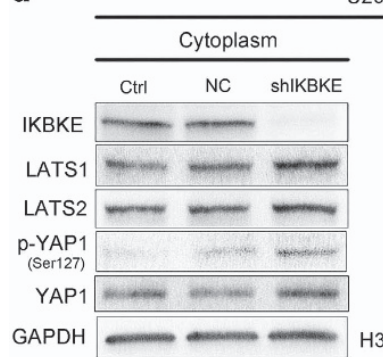

U251

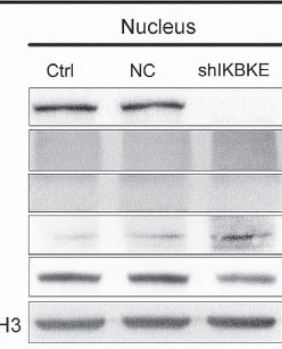

b

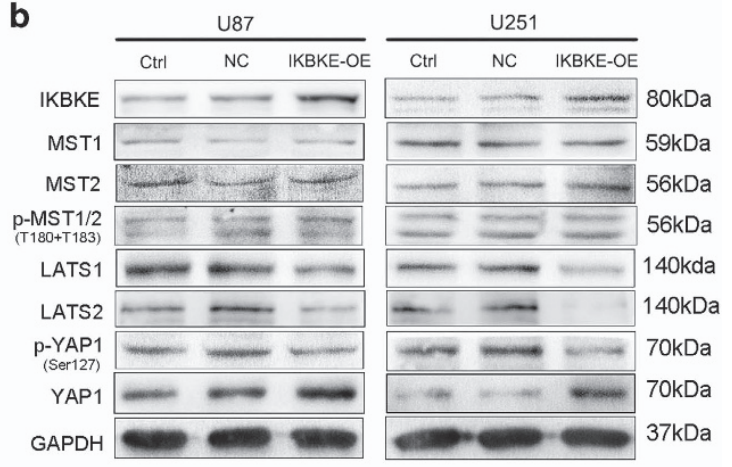

e

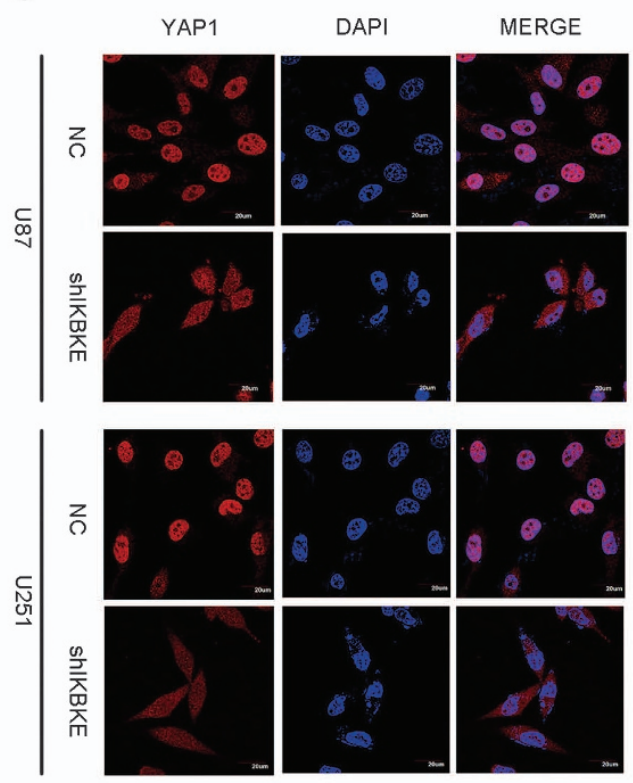

Figure 5 The Hippo pathway was involved in the proliferative regulation of glioma cells mediated by IKBKE. (a) U87 and U251 cells were transfected with control shRNA or IKBKE shRNA for $48 \mathrm{~h}$, and the effects of IKBKE knockdown on the protein expression of the Hippo pathway were determined by western blot. (b) Glioma cells were transfected with IKBKE-expressing plasmid for $48 \mathrm{~h}$, and the effects of IKBKE up-regulation on the protein expression were determined by western blot as in a. (c and d) Expression of YAP1 in the cytoplasmic and nuclear fractions was examined by western blot. (e) IKBKE-knockdown U87 and U251 cells were stained with DAPI and the antibody against YAP1 (scale bar $=20 \mu \mathrm{m})$

(Figures $7 \mathrm{a}$ and $\mathrm{b}$ ). Moreover, the tumor weight of mice treated with amlexanox was reduced by $50.9 \%$ compared with that of the control group (Figure 7c). In addition, no significant differences in body weights were observed between amlexanox treatment and the control group (Figure 7d). Amlexanox did not alter organs morphology nor induce hemorrhage in the heart, lung, kidney, spleen and liver (Supplementary Figures $4 \mathrm{a}$ and b).

To further confirm the therapeutic potential of amlexanox, orthotopic intracranial mouse models were constructed using the U87 glioma cell line, followed by amlexanox or DMSO treatment. The results of the bioluminescence imaging (BLI) showed that tumor growth was delayed in the amlexanox treatment group compared to that in the DMSO group (Figures 8a and b). Amlexanox-treated mice survived markedly longer than DMSO-treated mice. The results suggested that amlexanox could prolong the survival time of tumorbearing mice (Figure 8c). As shown in Figure 8d, IHC showed that the expression levels of IKBKE was significantly lower in the brain tumor tissue of amlexanox-treated mice. The results suggested that amlexanox had an effect on glioma cells in vivo by downregulating IKBKE. Moreover, the expression of LATS2 and $p$-YAP1(Ser127) increased, whereas YAP1, Axl, c-Myc, Cyr61, MMP-2 and MMP-9 expression levels were simultaneously decreased in amlexanox-treated group relative to the DMSO-treated group, which was consistent with the subcutaneous tumor tissues results (Figure 7e).

\section{Discussion}

IKBKE, which is a member of the IKK family, is known as a non-canonical IKK. Previous studies have illustrated that IKBKE plays a key role in inflammatory and metabolic 


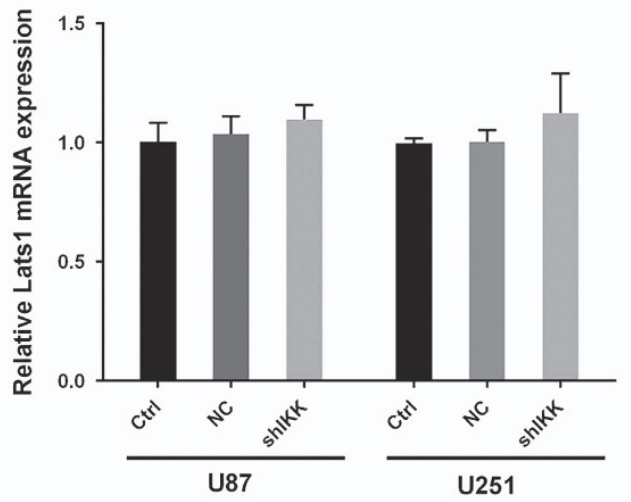

b

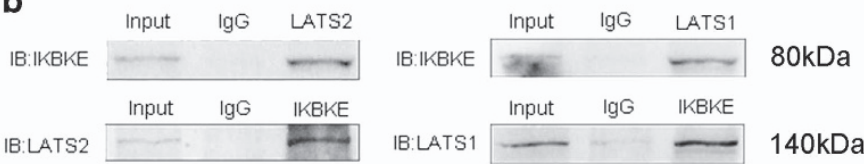

C

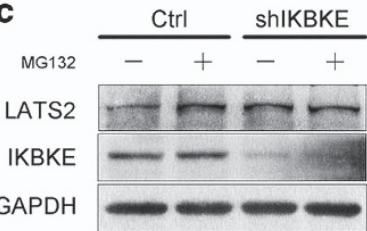

$140 \mathrm{kDa}$

$80 \mathrm{kDa}$

$37 \mathrm{kDa}$

d
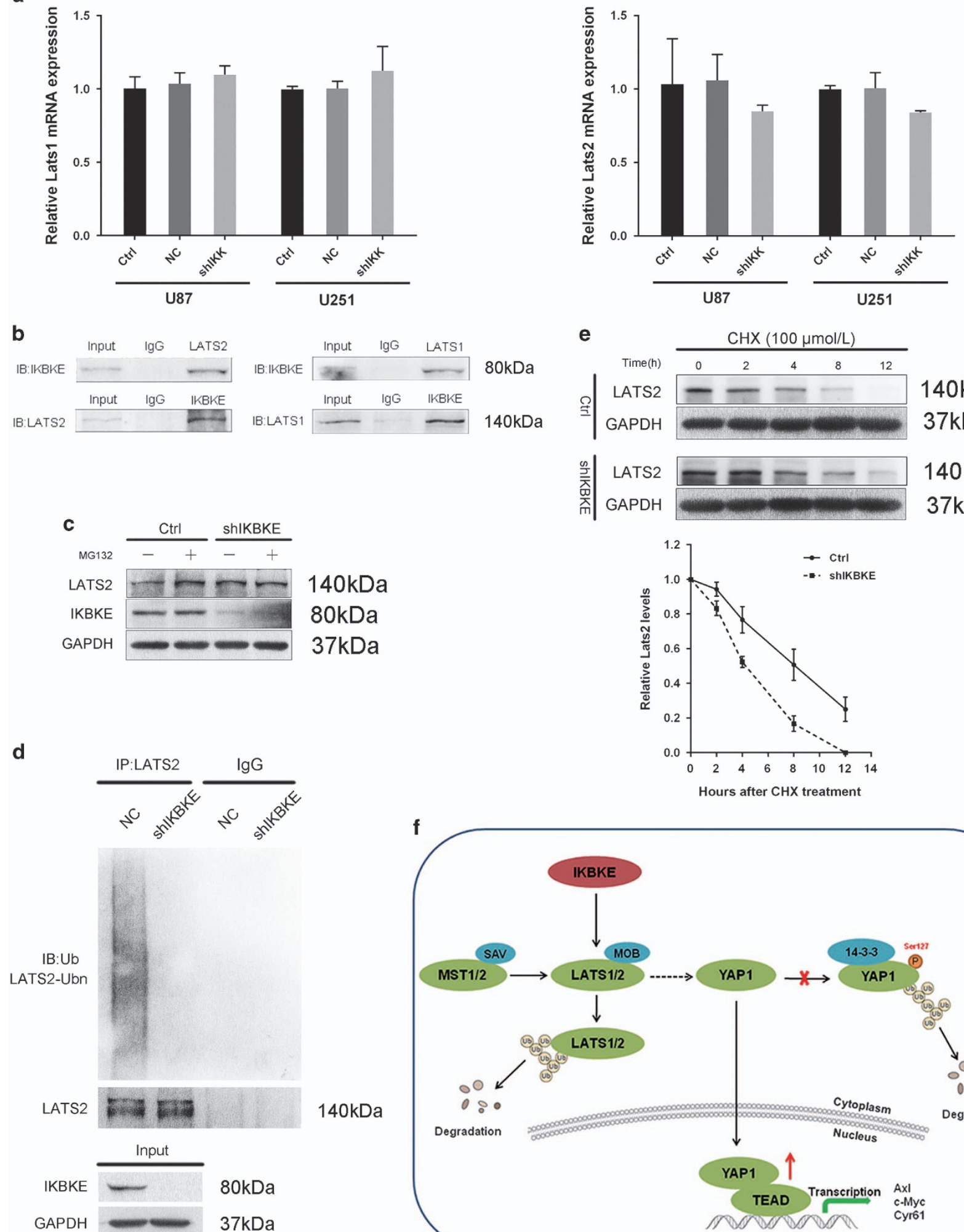

e

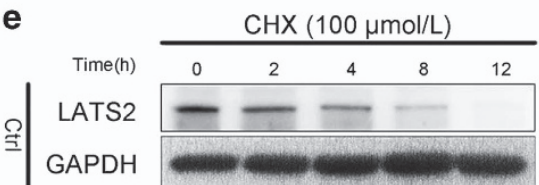

$140 \mathrm{kDa}$

LATS2

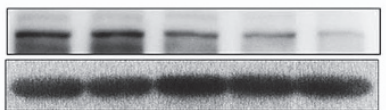

$37 \mathrm{kDa}$

䒽 $\mathrm{GAPDH}$

$140 \mathrm{kDa}$

$37 \mathrm{kDa}$
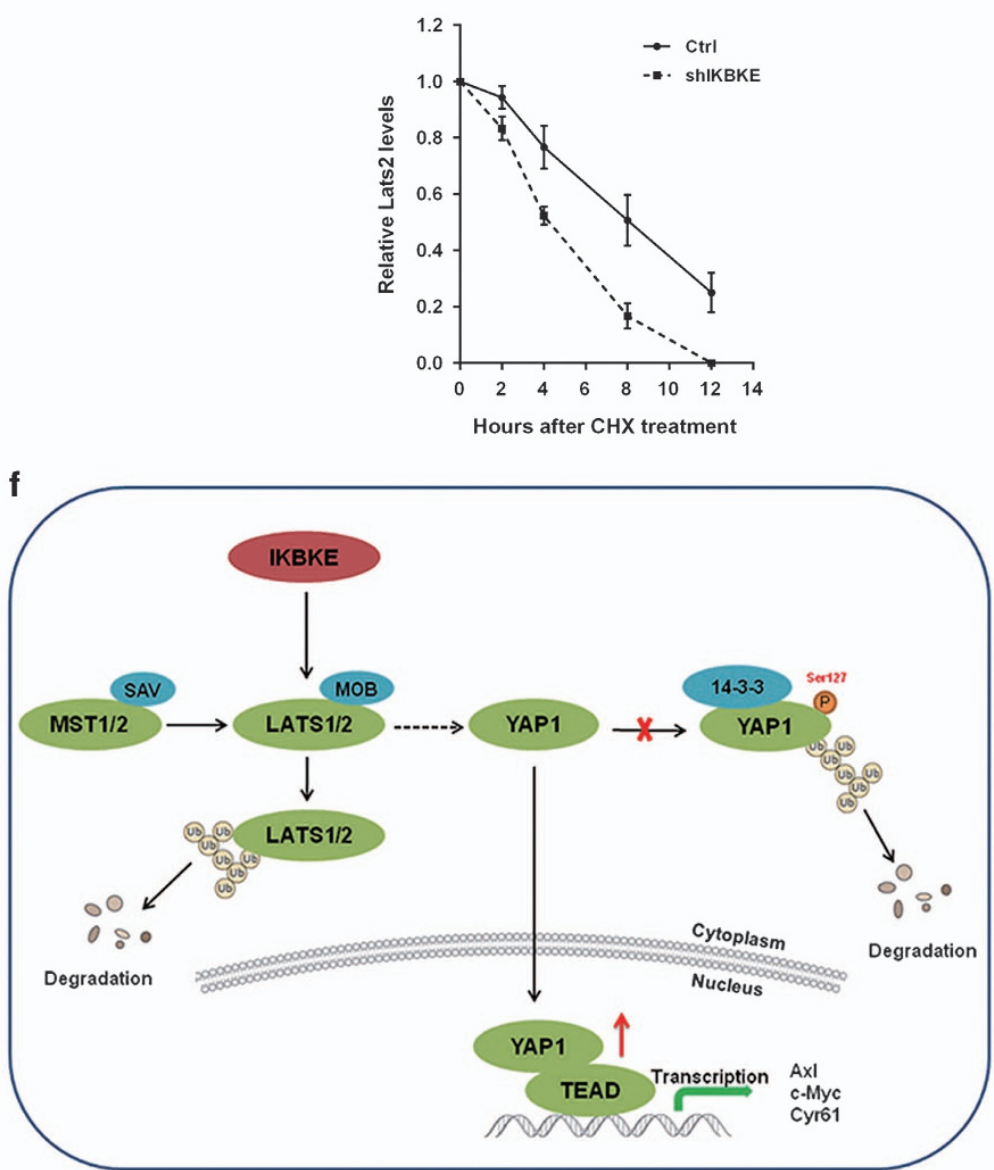

Figure 6 IKBKE interacts with and degrades LATS2. (a) Expression of LATS1/2 in IKBKE knockdown and control cells was examined at transcript levels. (b) The endogenous interaction between IKBKE and LATS1/2 in U87 cells was analyzed by immunoprecipitation. (c) LATS2 expression in IKBKE-knockdown U87 cells and control cells was measured after treatment with or without $20 \mu \mathrm{M}$ MG132 for $12 \mathrm{~h}$. (d) Decreased LATS2 ubiquitylation level by IKBKE knockdown in U87 cells. (e) Measurement of LATS2 in cell lysates harvested at 0, 2, 4, 8 and $12 \mathrm{~h}$ after the addition of $\mathrm{CHX}(100 \mu \mathrm{M})$ to arrest protein synthesis. (f) Schematic diagram of the mechanism of amlexanox-mediated antitumor activity by downregulation of IKBKE in GBM. IKBKE directly binds to and negatively regulates LATS1/2, which promotes YAP1 cytoplasmic retention and subsequent degradation 

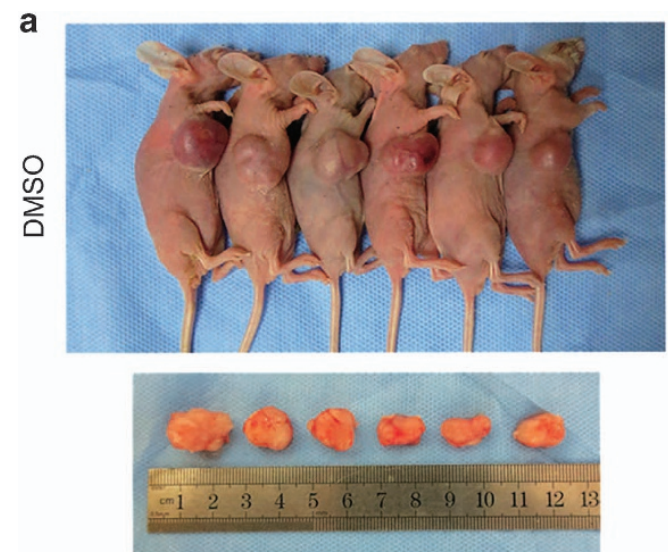

b

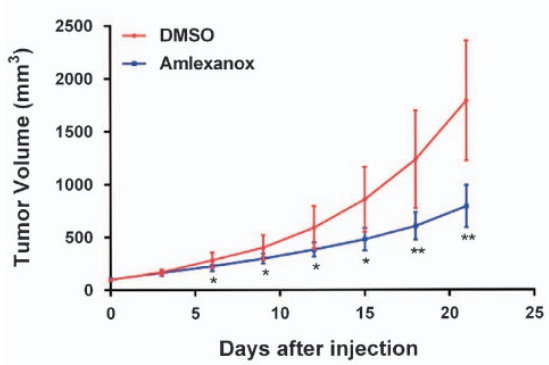

e

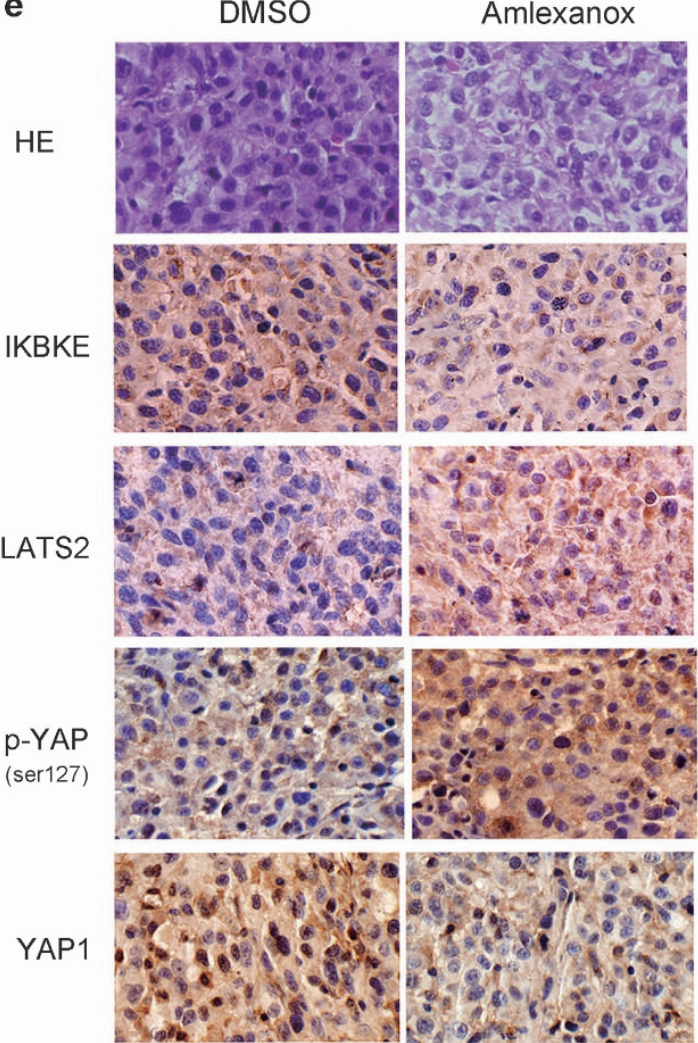

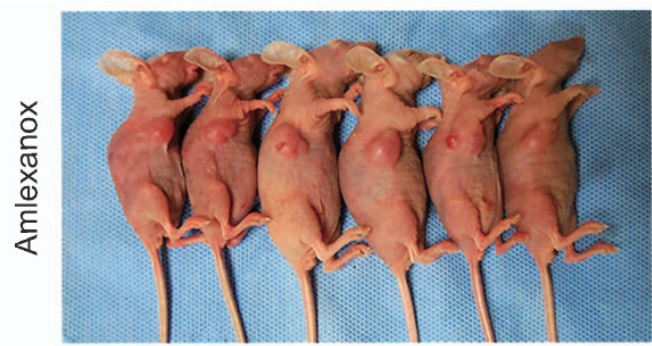

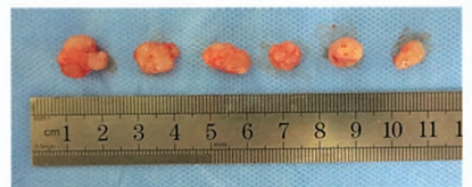

d

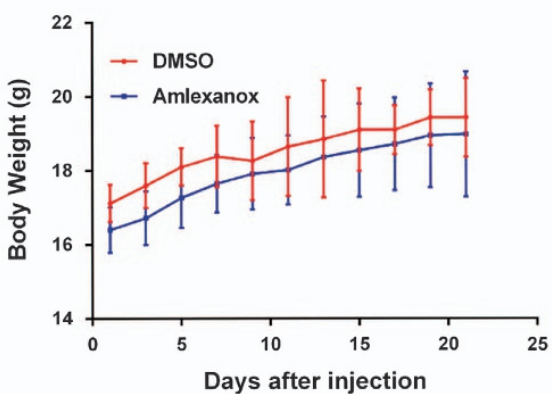

DMSO

Amlexanox

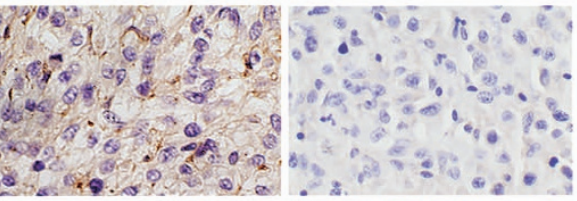

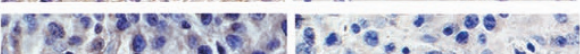

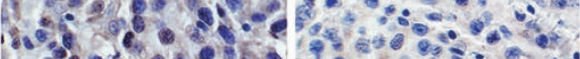

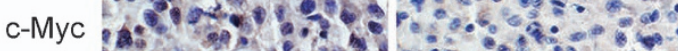

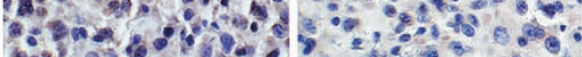

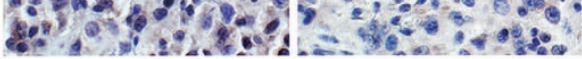

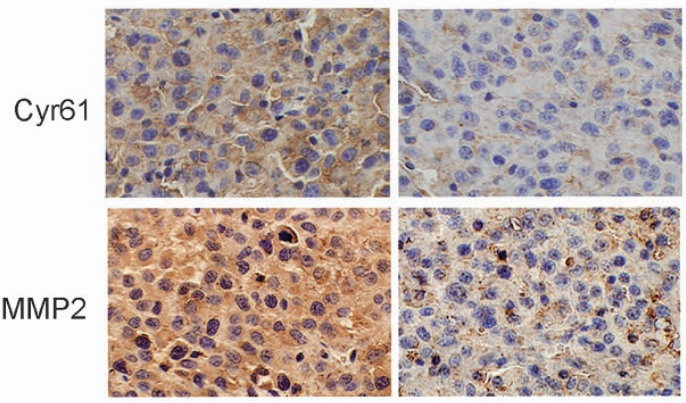

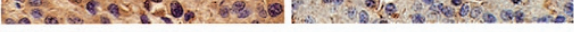

MMP9

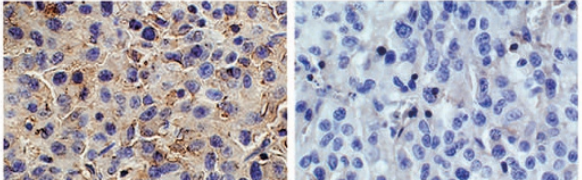


diseases. ${ }^{12,13,28-32}$ Recently, IKBKE has been implicated as an oncogenic protein and found to be upregulated/activated in multiple cancers. Over-expressing IKBKE can result in malignant transformation in various cell types. ${ }^{8}$ Thus, IKBKE has been considered as a promising therapeutic target for the treatment of cancer.

As a small molecular inhibitor of IKBKE, previous reports have illustrated that amlexanox has anti-inflammatory, antiallergic, immunomodulation activity, and it is used for treatment of aphthous ulcer, asthma, allergic rhinitis and obesity in the clinic. $^{12,33}$ In the present report, we showed that amlexanox displays anti-glioma properties in vitro with weak adverse effects on normal cells. Amlexanox is cytotoxic to glioma cells with IC50 values in the micromolar concentrations. Incubation of glioma cells with amlexanox inhibits cellular proliferation, migration and invasion and induces G0/ G1 phase arrest and apoptosis in U87 and U251 cells. In addition, amlexanox capable of directly binding to IKBKE and modulating its activity, and a reducing the protein levels of IKBKE have been reported. ${ }^{13,20}$ In contrast, the mRNA level of IKBKE was not decreased after amlexanox treatment, suggesting that amlexanox does not affect IKBKE transcription. Therefore, further studies are needed to demonstrate the mechanism by which amlexanox treatment induces downregulation of IKBKE protein levels.

Many previous studies have focused on downstream targets of IKBKE. IKBKE has been reported to activate the NF- $\kappa \mathrm{B}$ pathway by interacting with several cellular proteins, ${ }^{34-36}$ that induce expression of the pro-tumorigenic cytokines IL-6 and CCL5 and subsequent activation of the JAK/STAT pathway. ${ }^{37}$ In addition, IKBKE can activate AKT by directly phosphorylating AKT on Thr308 and Ser473 independent of mTORC2. ${ }^{38}$ IKBKE is also able to inhibit FOXO3a by inducing FOXO3a nuclearcytoplasmic translocation and protein degradation. ${ }^{39}$

The Hippo pathway can suppress cell proliferation and invasion, promote apoptosis and control organ size in diverse species, whereas pathway deregulation can induce tumorigenesis in model organisms and occurs in different kinds of human tumors, including lung, ovarian, colorectal and liver cancer. ${ }^{14}$ LATS1/2 could regulate cell migration through YAP and YAP-regulated transcriptional activity. ${ }^{40}$ YAP1 is a downstream transcriptional activator of the Hippo pathway, and is phosphorylated and inactivated by the pathway cascades. The loss of the Hippo pathway or aberrant activation of YAP1 will lead to nuclear accumulation of YAP1, thus promoting the expression of genes required for cell proliferation through direct binding to their cognate transcription factors. ${ }^{41}$

As a serine/threonine protein kinase, we speculate that IKBKE possibly participates in the regulation of the Hippo pathway, which is a kinase cascade and activation of LATS1/2 kinases (and inactivation of YAP/TAZ) represents the major functional output of the pathway. To confirm that amlexanox inhibited cell growth through the Hippo pathway activated by downregulation of IKBKE, we examined the protein levels of the Hippo pathway after amlexanox treatment. The results of showed that amlexanox could regulate LATS1/2 and YAP1 phosphorylation in a dose- and time-dependent manner. However, the protein levels of MST1/2 and the phosphorlevels of MST1/2 (Thr183 in MST1 and Thr180 in MST2), which could phosphorylate and activate LATS1/2, were not affected. Thus, we believe that the Hippo pathway might be one of the most important downstream pathways for the antitumor activity of amlexanox against GBM. To fully understand the possible mechanism by which IKBKE affects the Hippo pathway, we demonstrated that knockdown or over-expression of IKBKE affected the abundance of LATS1/2, phosphorylation status of YAP1 and the expression of Axl, c-Myc and Cyr61, which are the downstream targets of YAP1 by interaction with TEAs. ${ }^{21}$ However, we did not detect a significant change in MST1/2 and phospho-levels of MST1/2 (Thr183 in MST1 and Thr180 in MST2) upon IKBKE over-expression or depletion. Furthermore, we demonstrated, for the first time, that IKBKE directly bound to LATS1/2, and facilitated their polyubiquitin degradation. Meanwhile, our data showed that IKBKE did not alter mRNA levels of LATS1/2 in glioma cells. All of above data support the conclusion that IKBKE regulates the Hippo pathway through post-translational control of LATS1/2.

Amlexanox also exhibited promising antitumor efficacy in subcutaneous glioma xenograft models, which is consistent with its strong anti-proliferative activity in vitro. To verify whether amlexanox is able to cross the blood brain barrier (BBB), orthotopic intracranial mouse models were constructed. The data demonstrated that amlexanox not only significantly reduced brain tumor growth and the expression of IKBKE but also prolonged the survival of the intracranial models, suggesting a good BBB permeability of amlexanox.

In conclusion, our study is the first to demonstrate that amlexanox displays promising anticancer activity against glioma cells harboring high levels of IKBKE in vitro and in vivo. We further demonstrate a new role for IKBKE as a negative regulator of LATS $1 / 2$ and an inactivator of the Hippo pathway. However, it should be noted out that the effects on other IKBKE-regulated pathways may also contribute to the activity of amlexanox in glioma cells. These findings indicate that amlexanox is a promising chemotherapeutic compound for the treatment of GBM.

\section{Materials and Methods}

Cell lines and materials. Human glioma cell lines (U87 and U251) were purchased from the American Type Culture Collection (ATCC) (Manassas, VA, USA). The cells were cultured in Dulbecco's Modified Eagle's Medium (DMEM) (Gibco, USA) supplemented with $10 \%$ fetal bovine serum (FBS) (Gibco) and were incubated in a humidified atmosphere of $5 \% \mathrm{CO}_{2}$ at $37^{\circ} \mathrm{C}$. Amlexanox (HY-B0713) was purchased from MedChem Express (MCE) (Shanghai, China), and a $150 \mathrm{mM}$

Figure 7 Amlexanox exhibited significant antitumor effects against subcutaneous tumors in vivo. (a) Images of nude mice and tumors from the DMSO and amlexanox treatment groups. (b) The tumor volumes and (c) weights, and (d) the body weights of mice were evaluated using the in vivo proliferation assay. (e) Representative images of the $\mathrm{HE}$ and immunohistochemical staining for IKBKE and the proteins of the Hippo pathway in tumor sections (x200 magnification). IKBKE protein was effectively inhibited by amlexanox treatment. Besides, the expressions of LATS2 and p-YAP1(Ser127) increased, whereas YAP1, Axl, c-Myc, Cyr61, MMP-2 and MMP-9 expression levels were simultaneously decreased in amlexanox-treated group relative to the DMSO-treated group. Data are shown as the mean \pm S.D. ${ }^{\star} P<0.05,{ }^{\star \star} P<0.01$, compared to the control $(n=6)$ 
a
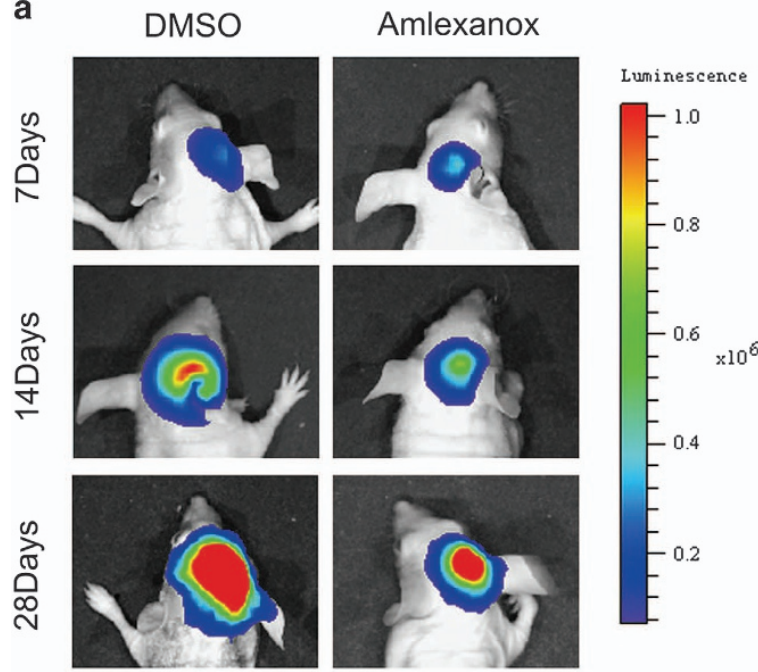

d<smiles>C1CCCCC1</smiles>

DMSO
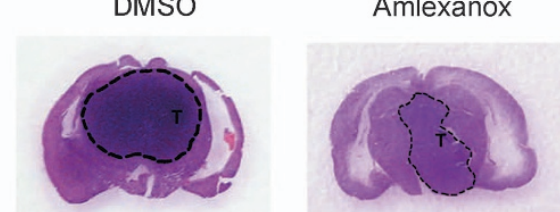

IKBKE

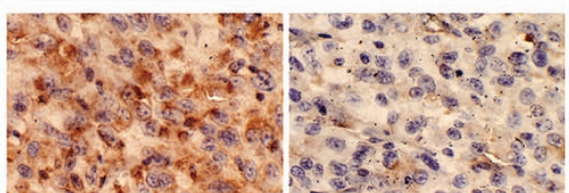

LATS2
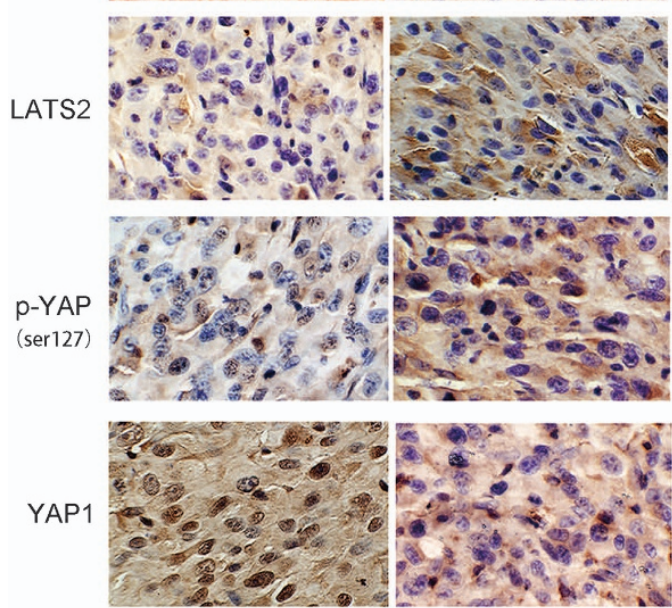

b

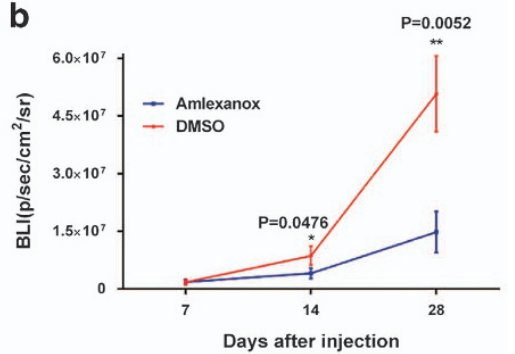

c

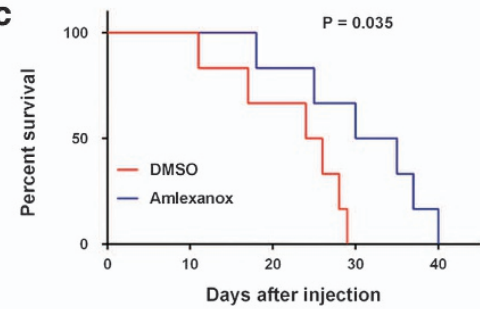

Axl

C-Myc

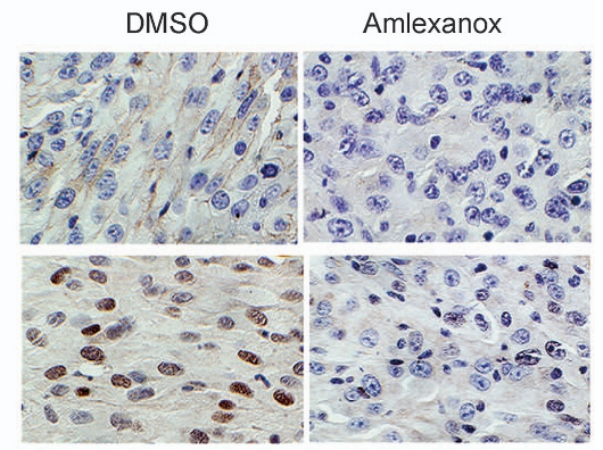

Cyr61

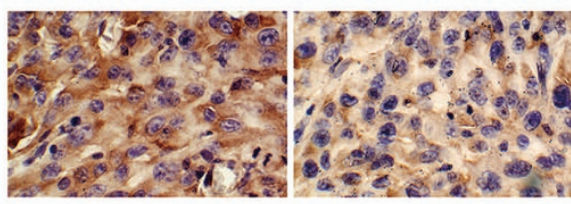

MMP2

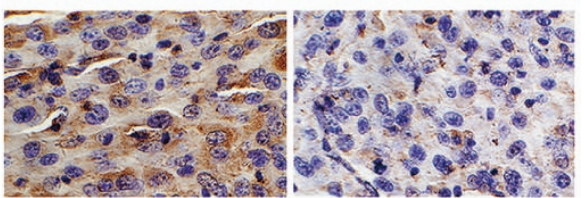

MMP9

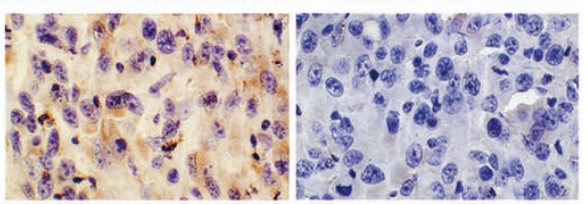

Figure 8 Antitumor effects of amlexanox in a U87 orthotopic intracranial model. The mice were treated with intraperitoneal injection with DMSO or amlexanox (100 mg/kg) daily. The treatment started from the 7th day after implantation and lasted for $\sim 21$ days. (a) Representative images of bioluminescence of mice on days 7,14 , and 28 after implantation. (b) Quantitative analysis of these bioluminescence images for the DMSO and amlexanox treatment groups. (c) The overall survival of mice in the DMSO and amlexanox treatment groups. There was a substantial survival benefit for the amlexanox-treated mice. (d) Representative images of the HE and immunohistochemical staining for IKBKE and the Hippo pathway proteins in tumor sections (×200 magnification). IKBKE protein was effectively inhibited by amlexanox treatment. Besides, the expressions of LATS2 and p-YAP1(Ser127) increased, whereas YAP1, Axl, c-Myc, Cyr61, MMP-2 and MMP-9 expression levels were simultaneously decreased in amlexanox-treated group relative to the DMSO-treated group. Data are shown as the mean \pm S.D. ${ }^{*} P<0.05,{ }^{* *} P<0.01$ compared to the control $(n=6)$

stock solution was prepared in dimethyl sulfoxide (DMSO) (Sigma Chemical, St. Louis, MO, USA). Antibodies against LATS1/2, MMP-2, MMP-9 and Cyr61 were purchased from Abcam (Shanghai, China). Antibodies against IKBKE, YAP1, p-YAP1 (Ser127), c-Myc and Ubiquitin were purchased from Cell Signaling Technology (Beverly, MA, USA). The Axl antibody was purchased from Bioss (Beijing, China).
CCK-8 cell viability assay. Cell viability was measured by a Cell Counting Kit-8 (CCK-8) assay (Dojindo, Japan). U87 and U251 cells $\left(4 \times 10^{3}\right.$ cells per well) were planted into 96-well plates and incubated for $12 \mathrm{~h}$. Then, the cells were treated with different concentrations of amlexanox $(0-300 \mu \mathrm{M})$. After incubation with amlexanox for $24,48,72$ or $96 \mathrm{~h}, \mathrm{CCK}-8$ was added to each well, and cells were incubated for $1.5 \mathrm{~h}$. The optical density was measured at $450 \mathrm{~nm}\left(\mathrm{OD}_{450}\right)$ using the 
microplate reader (Synergy2, BioTech, Vermont, VT, USA). Three independent experiments were performed.

Cell colony formation assay. U87 and U251 cells were seeded in six-well plates at a density of 2000 cells per well. After $12 \mathrm{~h}$ incubation, cultured cells were treated with various concentrations of amlexanox (50,100 and $150 \mu \mathrm{M})$. The culture medium was changed once every three days. The cells were grown for an additional 14 days. The cell colonies were fixed with methanol and stained with crystal violet. Colonies ( $\geqslant 50$ cells) were counted under an inverted microscope (Olympus, Japan). The rate of colony formation in each group was analyzed. Each experiment was performed in triplicate.

Cell cycle analysis. Glioma cells were seeded in six-well plates $\left(1 \times 10^{5}\right.$ cells per well). The cells were treated with DMSO or amlexanox for $72 \mathrm{~h}$ and then, harvested by trypsinization without EDTA. The cells were fixed with ice-cold $70 \%$ ethanol and incubated at $4{ }^{\circ} \mathrm{C}$ overnight. Then, the cells were stained for total DNA content with RNase (BD Biosciences, USA) and propidium iodide ( $\mathrm{PI}, \mathrm{BD}$ Biosciences). The samples were detected using a FACScan flow cytometer (BD Biosciences), and cell cycle was analyzed using FlowJo software V 7.6.

Cell apoptosis analysis. As described above, glioma cells were treated with DMSO or amlexanox. Then, the cells were stained with Annexin V-FITC and PI according to the manufacturer's instructions of the Annexin V-FITC Apoptosis Detection kit (KeyGEN BioTECH, Nanjing, China). Cell apoptosis was analyzed using a FACScan flow cytometer (BD Biosciences), and the data were analyzed using FlowJo software.

Scratch wound healing assay. U87 and U251 cells were pre-treated with amlexanox for $72 \mathrm{~h}$. Then, the cells were seeded in six-well plates $\left(5 \times 10^{5}\right.$ cells per well) until they reached $80-90 \%$ confluence. The scratch wounds were created in the monolayer of confluent glioma cells with a $200 \mu \mathrm{l}$ pipette tip. Then, these cells were incubated at $37{ }^{\circ} \mathrm{C}$ and in $5 \% \mathrm{CO}_{2}$. Wound healing was measured and recorded photographically over time using a phase-contrast microscope at 0,18 and $36 \mathrm{~h}$.

In vitro migration assay. The migratory capacity of glioma cells was evaluated using transwell filters ( $8 \mu \mathrm{m}$ pore size, Corning Costar, NY, USA). U87 and U251 cells were pre-treated with amlexanox for $72 \mathrm{~h}$. The cells were resuspended in serum-free DMEM at a density of $25 \times 10^{5}$ per ml, and $200 \mu \mathrm{l}$ of glioma cell suspension was added into the upper chambers. Then, $500 \mu \mathrm{l}$ of DMEM supplemented with $10 \%$ FBS was placed in the lower chambers. After $12 \mathrm{~h}$ or $24 \mathrm{~h}$ of incubation, the cells that had migrated to the underside surface were fixed with paraformaldehyde and stained with crystal violet. The average number of migratory cells was counted under an inverted microscope (Olympus Corp., Japan) over five random fields in each well. All experiments were performed in triplicate.

In vitro invasion assay. The effects of amlexanox on the invasion of glioma cells were elevated using a transwell invasion assay with inserts $(8 \mu \mathrm{m}$ pore size). The membranes of the transwell inserts were coated with Matrigel (BD Bioscience) and diluted with serum-free DMEM at a ratio of 1:3. U87 and U251 cells were prepared as described above. After $24 \mathrm{~h}$ or $48 \mathrm{~h}$ of incubation, the inserts were taken out and prepared for observation under an inverted microscope as described above. All tests were performed in triplicate.

Construction of the lentiviral vector system, transfection and immunofluorescence staining. A lentivirus-mediated short hairpin RNA (shRNA) was designed to knockdown the expression of IKBKE in glioma cells. The target sequence of the IKBKE shRNA was $5^{\prime}$-GCA TCA TCG AAC GGC TAA ATA-3', and a scrambled shRNA sequence of $5^{\prime}$-TTC TCC GAA CGT GTC ACG TTTC-3' served as a negative control. These shRNAs were ligated into the pFH-L vector (GeneChem, Shanghai, China) containing green fluorescent protein (GFP). The constructed lentiviral vectors were transfected into U87 and U251 cells according to the manufacturer's instructions. Successful infection was observed under a fluorescence microscope by the presence of green fluorescence in cells due to GFP. For immunofluorescence analysis, U87 and U251 cells were transfected with lentiviral vectors to detect the distribution of IKBKE, and YAP1 in the cytoplasm and nucleus, and the images were captured with an FV-500 laserscanning confocal microscope (Olympus, Japan).
Overexpression of IKBKE. The IKBKE and control vector plasmids were obtained from Addgene (Cambridge, MA, USA). The plasmids were transfected into U87 and U251 cells by using Lipofectamine 3000 (Invitrogen, USA) according to the manufacturer's protocol.

Western blot analysis, co-immunoprecipitation assays and ubiquitination assay. Glioma cells were treated with amlexanox (50, 100 and $150 \mu \mathrm{M}$ ) for $72 \mathrm{~h}$ or treated with $150 \mu \mathrm{M}$ amlexanox for 24,48 and $72 \mathrm{~h}$. Cultured cells were lysed in RIPA buffer supplemented with protease inhibitor. The protein concentrations were evaluated with a BCA protein assay kit (Pierce, USA). The samples were heated in $100^{\circ} \mathrm{C}$ water for $10 \mathrm{~min}$ to denature the proteins, separated on 10 or $12 \%$ SDS-PAGE gels, and electrophoretically transferred to a PVDF membrane (Millipore, USA). For the co-immunoprecipitation (Co-IP) experiments, glioma cells were lysed in IP Lysis/Wash Buffer (Thermo, USA). Fifty microliters of protein G agarose (Santa Cruz Biotechnology, CA, USA) was mixed with a specific monoclonal antibody or a nonspecific lgG overnight at $4{ }^{\circ} \mathrm{C}$. The immunoprecipitated proteins were separated by SDS-PAGE and analyzed by western blot. For endogenous LATS2 ubiquitination assay, Lysates were incubated using $2 \mu \mathrm{g}$ anti-LATS2 antibody and analyzed by western blot using anti-Ubiquitin or anti-LATS2 antibodies.

qRT-PCR analyses. Total RNA was prepared from glioma cells by using the Trizol reagent (Invitrogen) according to the manufacturer's instructions. Reverse transcription was performed using the GoScript Reverse Transcriptase kit (Promega, Madison, WI, USA). qRT-PCR was carried out as follows: $10 \mathrm{~min}$ at $95{ }^{\circ} \mathrm{C}$ followed by 40 cycles of $15 \mathrm{~s}$ at $95{ }^{\circ} \mathrm{C}$ and $40 \mathrm{~s}$ at $55^{\circ} \mathrm{C}$. The primer sequences used for qRT-PCR are shown in Supplementary Table 1.

Nude mouse model. To evaluate whether amlexanox inhibits tumor growth in vivo, the U87 cell line was selected to establish a nude mouse model. Female BALB/ c nude mice (3-4 weeks, 11-14 g) were purchased from the Animal Center of the Cancer Institute at Chinese Academy of Medical Science (Beijing, China). To establish subcutaneous model, $5 \times 10^{6} \mathrm{U} 87$ cells in $200 \mu$ l serum-free DMEM were injected into the right hind flank region of each mouse. After the tumors reached a volume of $100 \mathrm{~mm}^{3}$, the nude mice were randomized into two groups ( $n=6$ per group). Then, amlexanox (100 mg/kg/day) or DMSO was injected intraperitoneally into the mice everyday for three weeks. The tumor volume was measured every 3 days using a digital caliper and calculated as follows: volume $\left(\mathrm{mm}^{3}\right)=$ length $\times$ width ${ }^{2} / 2$. At the end of the experiment, all mice were anesthetized and sacrificed, and tumors were weighed. To establish an intracranial model, $5 \times 10^{4}$ U87 cells with a luciferase-encoding lentivirus were injected into the mice ( $n=6$ per group) stereotactically. Luciferaseencoding lentivirus were purchased from GeneChem (Shanghai, China). The lentivirus vector is GV260: Ubi-MCS-Luc-IRES-Puromycin. After 7 days post implantation, the mice were injected intraperitoneally with amlexanox (100 mg/kg/day) or DMSO everyday during the survival period. Intracranial tumor growth was detected by using BLI on days 7, 14 and 28, using the IVIS Spectrum Live Imaging System (PerkinEImer, Branford, USA). The animal research was performed according to the internationally recognized guidelines and national regulations. Subcutaneous tumors and brains were extracted and fixed in $10 \%$ formalin and then, embedded in paraffin for $\mathrm{HE}$ and $\mathrm{IHC}$.

Statistical analysis. All results were expressed as the mean \pm S.D. Statistical analysis was performed with SPSS 16 software. The differences between two groups were assessed with a Student's $t$-tests and differences between multiple groups were assessed using a one-way analysis of variance (ANOVA) test followed by Tukey's post hoc test. $P<0.05$ was considered to be statistically significant.

\section{Conflict of Interest}

The authors declare no conflict of interest.

Acknowledgements. This work was supported by the National Natural Science Foundation of China (Grant No. 81572490).

\section{Publisher's Note}

Springer Nature remains neutral with regard to jurisdictional claims in published maps and institutional affiliations.

1. Lu L, Zhang S, Li C, Zhou C, Li D, Liu P et al. Cryptotanshinone inhibits human glioma cell proliferation in vitro and in vivo through SHP-2-dependent inhibition of STAT3 activation. Cell Death Dis 2017; 8: e2767. 
2. Li H, Chen L, Zhang A, Wang G, Han L, Yu K et al. Silencing of IKKepsilon using siRNA inhibits proliferation and invasion of glioma cells in vitro and in vivo. Int J Oncol 2012; 41 169-178.

3. Siebzehnrubl FA, Reynolds BA, Vescovi A, Steindler DA, Deleyrolle LP. The origins of glioma: E Pluribus Unum? Glia 2011; 59: 1135-1147.

4. Lima FR, Kahn SA, Soletti RC, Biasoli D, Alves T, da Fonseca AC et al. Glioblastoma: therapeutic challenges, what lies ahead. Biochim Biophys Acta 2012; 1826: 338-349.

5. Stupp R, Hegi ME, Mason WP, van den Bent MJ, Taphoorn MJ, Janzer RC et al. Effects of radiotherapy with concomitant and adjuvant temozolomide versus radiotherapy alone on survival in glioblastoma in a randomised phase III study: 5 -year analysis of the EORTCNCIC trial. Lancet Oncol 2009; 10: 459-466.

6. Ostrom QT, Gittleman H, Fulop J, Liu M, Blanda R, Kromer C et al. CBTRUS statistical report: primary brain and central nervous system tumors diagnosed in the United States in 2008-2012. Neuro-oncology 2015; 17: iv1-iv62.

7. Argyriou AA, Kalofonos HP. Molecularly targeted therapies for malignant gliomas. Mol Med 2009; 15: 115-122.

8. Boehm JS, Zhao JJ, Yao J, Kim SY, Firestein R, Dunn IF et al. Integrative genomic approaches identify IKBKE as a breast cancer oncogene. Cell 2007; 129: 1065-1079.

9. Guo JP, Shu SK, He L, Lee YC, Kruk PA, Grenman S et al. Deregulation of IKBKE is associated with tumor progression, poor prognosis, and cisplatin resistance in ovarian cancer. Am J Pathol 2009; 175: 324-333.

10. Peant B, Diallo JS, Dufour F, Le Page C, Delvoye N, Saad F et al. Over-expression of IkappaB-kinase-epsilon (IKKepsilon/IKKi) induces secretion of inflammatory cytokines in prostate cancer cell lines. Prostate 2009; 69: 706-718.

11. Guan $\mathrm{H}$, Zhang $\mathrm{H}$, Cai J, Wu J, Yuan J, Li J et al. IKBKE is over-expressed in glioma and contributes to resistance of glioma cells to apoptosis via activating NF-KB. J Pathol 2011; 223: 436-445.

12. Zhang Y, Guan H, Li J, Fang Z, Chen W, Li F. Amlexanox suppresses osteoclastogenesis and prevents ovariectomy-induced bone loss. Sci Rep 2015; 5: 13575.

13. Reilly SM, Chiang SH, Decker SJ, Chang L, Uhm M, Larsen MJ et al. An inhibitor of the protein kinases TBK1 and IKK-varepsilon improves obesity-related metabolic dysfunctions in mice. Nat Med 2013; 19: 313-321.

14. Harvey KF, Zhang X, Thomas DM. The Hippo pathway and human cancer. Nat Rev Cancer 2013; 13: 246-257.

15. Varelas $X$. The Hippo pathway effectors TAZ and YAP in development, homeostasis and disease. Development 2014; 141: 1614-1626.

16. Hansen CG, Moroishi T, Guan KL. YAP and TAZ: a nexus for Hippo signaling and beyond. Trends Cell Biol 2015; 25: 499-513.

17. Dong J, Feldmann G, Huang J, Wu S, Zhang N, Comerford SA et al. Elucidation of a universal size-control mechanism in Drosophila and mammals. Cell 2007; 130: 1120-1133.

18. Orr BA, Bai H, Odia Y, Jain D, Anders RA, Eberhart CG. Yes-associated protein 1 is widely expressed in human brain tumors and promotes glioblastoma growth. $J$ Neuropathol Exp Neurol 2011; 70: 568-577.

19. Forsyth PA, Wong H, Laing TD, Rewcastle NB, Morris DG, Muzik $\mathrm{H}$ et al. Gelatinase-A (MMP-2), gelatinase-B (MMP-9) and membrane type matrix metalloproteinase-1 (MT1MMP) are involved in different aspects of the pathophysiology of malignant gliomas. $\mathrm{Br} \mathrm{J}$ Cancer 1999; 79: 1828-1835.

20. Challa S, Guo JP, Ding X, Xu CX, Li Y, Kim D et al. IKBKE is a substrate of EGFR and a therapeutic target in non-small cell lung cancer with activating mutations of EGFR. Cancer Res 2016; 76: 4418-4429.

21. Zhou Y, Huang T, Cheng AS, Yu J, Kang W, To KF. The TEAD family and its oncogenic role in promoting tumorigenesis. Int $\mathrm{J} \mathrm{Mol} \mathrm{Sci} \mathrm{2016;} \mathrm{17:} \mathrm{E138.}$

22. Ma B, Cheng H, Gao R, Mu C, Chen L, Wu S et al. Zyxin-Siah2-Lats2 axis mediates cooperation between Hippo and TGF-beta signalling pathways. Nat Commun 2016; 7 11123.

23. Zhao B, Wei X, Li W, Udan RS, Yang Q, Kim J et al. Inactivation of YAP oncoprotein by the Hippo pathway is involved in cell contact inhibition and tissue growth control. Genes Dev 2007; 21: 2747-2761.

24. Hao Y, Chun A, Cheung K, Rashidi B, Yang X. Tumor suppressor LATS1 is a negative regulator of oncogene YAP. J Biol Chem 2008; 283: 5496-5509.
25. Xiao L, Chen Y, Ji M, Dong J. KIBRA regulates Hippo signaling activity via interactions with large tumor suppressor kinases. J Biol Chem 2011; 286: 7788-7796.

26. Ma B, Chen Y, Chen L, Cheng H, Mu C, Li J et al. Hypoxia regulates Hippo signalling through the SIAH2 ubiquitin E3 ligase. Nat Cell Biol 2015; 17: 95-103.

27. Yabuta N, Fujii T, Copeland NG, Gilbert DJ, Jenkins NA, Nishiguchi H et al. Structure, expression, and chromosome mapping of LATS2, a mammalian homologue of the Drosophila tumor suppressor gene lats/warts. Genomics 2000; 63: 263-270.

28. Corr M, Boyle DL, Ronacher L, Flores N, Firestein GS. Synergistic benefit in inflammatory arthritis by targeting I kappaB kinase epsilon and interferon beta. Ann Rheum Dis 2009; 68: 257-263.

29. Sandling JK, Garnier S, Sigurdsson S, Wang C, Nordmark G, Gunnarsson I et al. A candidate gene study of the type I interferon pathway implicates IKBKE and IL8 as risk loci for SLE. Eur J Hum Genet 2011; 19: 479-484.

30. Bulek K, Liu C, Swaidani S, Wang L, Page RC, Gulen MF et al. The inducible kinase IKKi is required for IL-17-dependent signaling associated with neutrophilia and pulmonary inflammation. Nat Immunol 2011; 12: 844-852.

31. Moser CV, Kynast K, Baatz K, Russe OQ, Ferreiros N, Costiuk H et al. The protein kinase IKKepsilon is a potential target for the treatment of inflammatory hyperalgesia. $J$ immunol 2011; 187: 2617-2625

32. Scheja L, Heese B, Seedorf K. Beneficial effects of IKKepsilon-deficiency on body weight and insulin sensitivity are lost in high fat diet-induced obesity in mice. Biochem Biophys Res Commun 2011; 407: 288-294.

33. Reilly SM, Ahmadian M, Zamarron BF, Chang L, Uhm M, Poirier B et al. A subcutaneous adipose tissue-liver signalling axis controls hepatic gluconeogenesis. Nat Commun 2015; 6: 6047.

34. Adli M, Baldwin AS. IKK-i//KKepsilon controls constitutive, cancer cell-associated NF-KB activity via regulation of Ser-536 p65/RelA phosphorylation. J Biol Chem 2006; 281: 26976-26984.

35. Harris J, Oliere $S$, Sharma $S$, Sun $Q$, Lin R, Hiscott $J$ et al. Nuclear accumulation of cRel following C-terminal phosphorylation by TBK1/IKK. J Immunol 2006; 177: 2527-2535.

36. Shen RR, Zhou AY, Kim E, Lim E, Habelhah H, Hahn WC. IkappaB kinase epsilon phosphorylates TRAF2 to promote mammary epithelial cell transformation. Mol Cell Biol 2012; 32: 4756-4768.

37. Barbie TU, Alexe G, Aref AR, Li S, Zhu Z, Zhang X et al. Targeting an IKBKE cytokine network impairs triple-negative breast cancer growth. J Clin Investig 2014; 124: 5411-5423.

38. Xie X, Zhang D, Zhao B, Lu MK, You M, Condorelli G et al. IkappaB kinase epsilon and TANK-binding kinase 1 activate AKT by direct phosphorylation. Proc Natl Acad Sci USA 2011; 108: 6474-6479.

39. Guo JP, Tian W, Shu S, Xin Y, Shou C, Cheng JQ. IKBKE phosphorylation and inhibition of FOXO3a: a mechanism of IKBKE oncogenic function. PLOS ONE 2013; 8: e63636.

40. Zhang J, Smolen GA, Haber DA. Negative regulation of YAP by LATS1 underscores evolutionary conservation of the Drosophila Hippo pathway. Cancer Res 2008; 68: 2789-2794.

41. Wang H, Du YC, Zhou XJ, Liu H, Tang SC. The dual functions of YAP-1 to promote and inhibit cell growth in human malignancy. Cancer Metastasis Rev 2014; 33: 173-181.

Cell Death and Disease is an open-access journal published by Nature Publishing Group. This work is licensed under a Creative Commons Attribution 4.0 International License. The images or other third party material in this article are included in the article's Creative Commons license, unless indicated otherwise in the credit line; if the material is not included under the Creative Commons license, users will need to obtain permission from the license holder to reproduce the material. To view a copy of this license, visit http://creativecommons.org/licenses/by/4.0/

(C) The Author(s) 2017

Supplementary Information accompanies this paper on Cell Death and Disease website (http://www.nature.com/cddis) 